\title{
A variational approach to denoising problem
}

\author{
Dang Ngoc Hoang Thanh \\ Department of Information Technology, Hue Industrial College, 70 Nguyen Hue st., Hue city, Vietnam \\ Advisors/s: Dvoenko Sergey \\ Date and location of PhD thesis defense: 29 June 2016, Tula State University, Tula city, Russia.
}

Received 7th September 2016; accepted 8th September 2016

\begin{abstract}
In many problems of image processing, digital images are always offered in the form of mathematical function. We call this function as a brightness or an intensity function. Such functions can be considered in the continuous or discrete forms. A digital image can be created by different digital devices, such as digital cameras, X-ray scanners, etc. In practice, such devices can give unexpected defects, for example, noise.

One of important problems of image processing is the denoising problem [1-3]: it needs to reconstruct the original version of a corrupted initial image given. This problem has many applications in many fields. The main goal of reconstructing is an image quality improvement based on image processing by specially developed denoising algorithms.

Many different types of noises can arise during the process of image formation in the form of signal-dependent or signal-independent noises [4]. The signal-dependent noise can be usually approximated by Poisson distribution (we call it Poisson noise). The signal-independent noise can be approximated by Gaussian distribution (we call it Gaussian noise). The Gaussian noise appears in almost types of digital images, and the Poisson noise, for example, in X-ray images. The problem of removing these noises is actual today.

When we remove noise in digital images, we usually prefer the Gaussian noise and omit other types of noise. Many methods have been developed in order to remove the Gaussian noise, such as: median filter [5], Wiener filter [6], ROF-model [7], etc. However, the Poisson noise is important too. Its variance is not the constant, but it depends on the intensity value of pixels. Many methods have been developed in order to remove this noise too, such as nonlocal PCA [8], ICA domain [9], Anscombe transform [10], modified ROF-model [11], etc.

Real noises can be effectively represented by the mixed Poisson-Gaussian noise [12]. This mixed noise usually appears in electronic microscopic images, in aerospace images, etc.

Hence, the problem of the mixed noise removing is the actual problem too. Theoretical models to solve this problem are based on the mixed Poisson-Gaussian noise distribution. In order to remove this noise, there are many methods, such as: multiscale gradient (Benvenuto) [13], alternative minimization (Gil-Rodrigo) [14], PURE-LET [4], generalized Anscombe transform based method [15], etc.

Theoretical models to remove the mixed noise usually are complicated ones. Such complexity is usually caused by multiple parameters of such models. Therefore, the performance of processing procedures is reduced, if the estimations of these parameters are not sufficiently optimal. Therefore, the problem to develop the mixed denoising
\end{abstract}

Correspondence to: dnhthanh@hueic.edu.vn

Recommended for acceptance by David Vázquez Bérmudez

DOI http://dx.doi.org/10.5565/rev/elcvia.991

ELCVIA ISSN: 1577-5097

Published by Computer Vision Center / Universitat Autonoma de Barcelona, Barcelona, Spain 
model with the small number of parameters to simplify a processing procedure and to increase the processing performance is actual right now.

In this dissertation we solve the problem of the mixed noise removal by using total variation, where the components of the mixed noise are given. Then we have to define the proportion of every component in the mixed noise. We call this noise linear combination of noises. Therefore, we consider the problem of linear combination of Gaussian and Poisson noises removal. Besides, we also consider this problem for the Gaussian noise and Poisson noise separately.

The variational approach is well-known and very promising. This concept was pioneered by Rudin. He proposed the ROF model [7]. The ROF model is used to remove only Gaussian noise. However, another important type like Poisson noise is usually presented in medical X-ray images. In order to remove this noise, Le T. developed so called modified ROF model [11]. Nevertheless, ROF and modified ROF models ineffectively treat this combination. ROF model gives priority to Gaussian noise, but modified ROF model gives it to Poisson noise.

Our goal is to combine ROF model (for Gaussian noise) and modified ROF model (for Poisson noise) to create new model that can treat this combination effectively. Our model will treat this combination with considering proportion of noise between them.

The difference of proposed approach from other known approaches is: the noise that we consider is not superposition, but linear combination of components of noises. This reason allows us obtain the simple model with small number of parameters and also obtain the simple algorithm to guaranty the high quality processing results.

\section{Experiment}

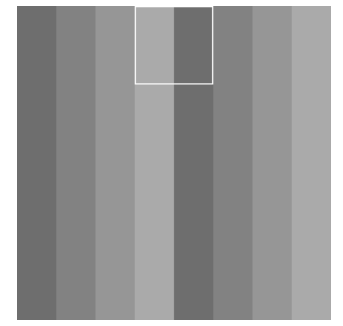

a)

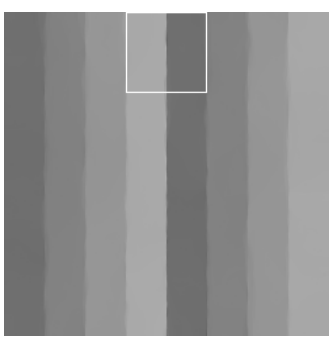

e)

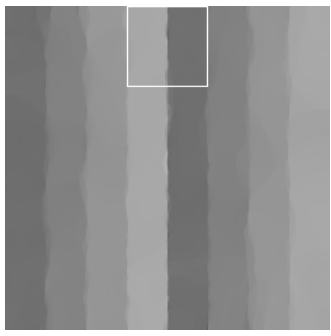

i)

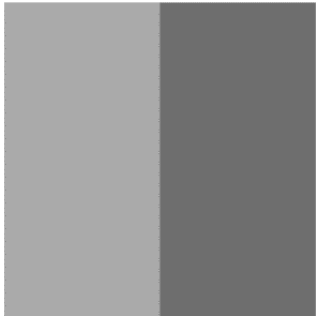

b)

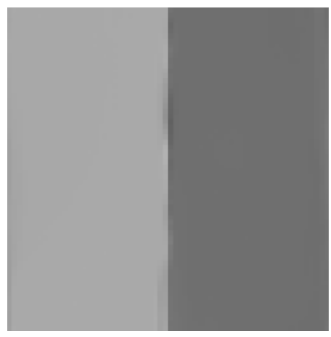

f)

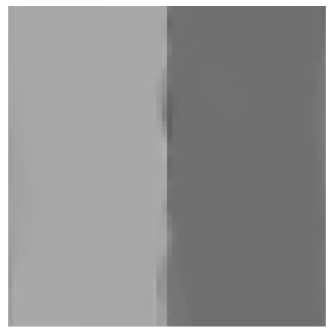

j)

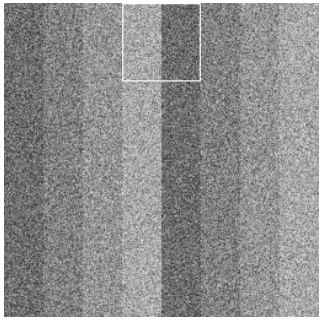

c)

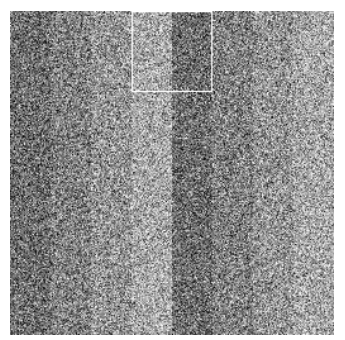

g)

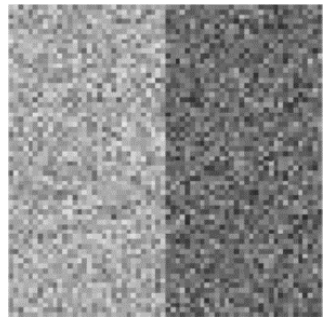

d)

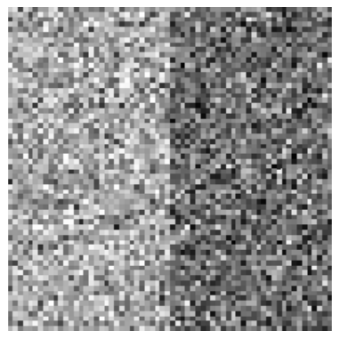

h)

Figure 1: Denoising of the artificial image: a)-b) original image, c)-d) noisy image with linear combination of noises, e)-f) denoised image (c), g)-h) noisy image with superposition of noises, i)-j) denoised image (g). 


\section{References}

[1] Micheal Elad. Sparse and Redundant Representations: Image denoising. Springer New York. 2010. P. 273-307.

[2] Gabriela Ghimpeteanu, Thomas Batard, Marcelo Bertalmio, Stacey Levine. Image and Signal Processing. Springer. Lecture Notes in Computer Science. 2014. V. 8509. P. 375-383.

[3] Qin Jia, ZhiQuan Li, XueFei Liu. Advances in Computer Science, Intelligent System and Environment. Springer. Series Advances in Intelligent and Soft Computing. 2011. V. 105. P. 303-308.

[4] F. Luisier, T. Blue, M. Unser. Image denoising in mixed Poisson-Gaussian noise. IEEE transaction on Image Processing. 2011. V. 20. N. 3. P. 696-708. doi:10.1109/tip.2010.2073477.

[5] Wang C. An improved adaptive median filter for Image denoising. ICCEE. 2012. V. 53 . N. 2.64. P. 393398.

[6] Abe C. Iterative Edge-Preserving adaptive Wiener filter for image denoising. ICCEE. 2012. V. 4. No. 4. P. 503-506. doi:10.7763/ijcee.2012.v4.543.

[7] Rudin L.I., Osher S., Fatemi E. Nonlinear total variation based noise removal algorithms. Physica D. 1992. V. 60. P. 259-268. doi:10.1016/0167-2789(92)90242-f.

[8] Joseph Salmon, Zachary Harmany, Charles-Alban Deledalle, Rebecca Willett. Poisson Noise Reduction with Non-local PCA. Journal of Mathematical Imaging and Vision. 2014. V. 48. Is. 2. P. 279-294. doi:10.1109/icassp.2012.6288081.

[9] Xian-Hua Han, Yen-Wei Chen, Zensho Nakao. An ICA-Based Method for Poisson Noise Reduction. Springer: series Lecture Notes in Computer Science. V. 2773. P. 1449-1454. doi:10.1007/978-3-54045224-9_195.

[10] F. Murtagh, J.-L. Starck, A. Bijaoui. Image restoration with noise suppression using a multiresolution support. Astron. Astrophys. Supplement Series. 1995. V. 112. P. 179-189.

[11] Le T., Chartrand R., Asaki T.J. A variational approach to reconstructing images corrupted by Poisson noise. Journal of mathematical imaging and vision. 2007. V. 27. I. 3. P. 257-263. doi:10.1007/s10851007-0652-y.

[12] Anna Jezierska, Caroline Chaux, Jean-Christophe Pesquet, and Hugues Talbot. An EM approach for Poisson-Gaussian noise modeling. Proceeding of the 19th European signal processing conference 2011. P. 2244-2248.

[13] F. Benvenuto, A. La Camera, C. Theys, A. Ferrari, H. Lanteri, and M. Bertero. The study of an iterative method for the reconstruction of images corrupted by Poisson and Gaussian noise. Inverse Problems. 2008. V. 24, Is. 3. P. 1-20. doi:10.1088/0266-5611/24/3/035016.

[14] Gil-Rodrigo, J. Portilla, D. Miraut, R. Suzrez-Mesa. Efficient joint Poisson-Gauss restoration using multiframe $l_{2}$ relaxed $l_{0}$ analysis-based sparsity. Proceeding of the 18th IEEE International Conference on Image Processing (ICIP). 2011. P. 1385-1388. doi:10.1109/icip.2011.6115697.

[15] M. Makitalo, A. Foi. Optimal inversion of the generalized Anscombe transformation for PoissonGaussian noise. IEEE transaction on Image Processing. 2013. V. 22. Is. 1. P. 91-103. doi:10.1109/tip.2012.2202675. 